\title{
A TEMÁTICA DAS BOAS PRÁTICAS OBSTÉTRICAS NO CONTEXTO DO PROJETO POLÍTICO-PEDAGÓGICO DA RESIDÊNCIA
}

\author{
Elisabete Mesquita Peres de Carvalho ${ }^{1}$, Leila Bernarda Donato Göttems ${ }^{2}$ e Dirce Bellezi \\ Guilhem 1 \\ 1Universidade de Brasília, Brasil. elisabete_mpc@yahoo.com.br; guilhem@unb.br \\ Escola Superior de Ciências da Saúde, Brasil. leila.gottems@gmail.com
}

\begin{abstract}
Resumo. Introdução: A educação e a saúde são espaços de produção de saberes destinados ao desenvolvimento humano. As mudanças na formação se operam de forma concomitante com a produção de muitos processos na gestão, no ensino, no serviço e na atenção. Objetivos: Verificar se os projetos pedagógicos dos cursos de residência obstétrica, de médicos (as) e enfermeiros (as), dos serviços de saúde da Secretaria de Saúde do Distrito Federal contemplam as recomendações das diretrizes nacionais e internacionais de atenção ao parto normal. Métodos: Estudo descritivo, exploratório, com triangulação de dados, de abordagem qualitativa, por meio de análise documental realizada em oito projetos pedagógicos e análise de conteúdo de entrevistas com 35 preceptores. Resultados: Apenas os projetos pedagógicos do curso de enfermagem contemplam textualmente as recomendações do Ministério da Saúde e da Organização Mundial de Saúde relacionadas às boas práticas obstétricas. Segundo os preceptores, eles buscam o alinhamento entre a teoria e a prática desde a sala de aula até os cenários de práticas. Conclusões: Sugere-se que os conteúdos dos projetos pedagógicos dos cursos da residência sejam revistos e atualizados, sempre que necessário, visando corroborar com a formação adequada dos futuros profissionais.
\end{abstract}

Palavras-chave: Formação Profissional; Projeto Político-Pedagógico; Residência; Parto Normal.

\section{THE THEME OF GOOD OBSTETRIC PRACTICES IN THE CONTEXT OF POLITICAL- PEDAGOGICAL PROJECT OF THE RESIDENCE}

\begin{abstract}
Introduction: Education and health are spaces for the production of knowledge for human development. The changes in training take place concurrently with the production of many processes in management, teaching, service and care. Objectives: To verify if the pedagogical projects of the obstetric residency courses, of doctors and nurses, of the health services of the Secretariat of Health of the Federal District include the recommendations of the national and international guidelines of care for normal childbirth. Methods: Descriptive, exploratory study, with data triangulation, with a qualitative approach, through documentary analysis carried out in eight pedagogical projects and content analysis of interviews with 35 preceptors. Results: Only the pedagogical projects of the nursing course textually contemplate the recommendations of the Ministry of Health and the World Health Organization related to good obstetric practices. According to the preceptors, they seek the alignment between theory and practice from the classroom to the practice scenarios. Conclusions: It is suggested that the contents of the pedagogical projects of the residency courses be reviewed and updated whenever necessary in order to corroborate the appropriate training of future professionals.
\end{abstract}

Keywords: Professional Training; Political-Pedagogical Project; Internship and Residency; Natural Childbirth.

\section{INTRODUÇÃO}

A educação e a saúde são espaços de produção de saberes destinados ao desenvolvimento humano. Há interseção nestes dois campos quando da construção de saberes e práticas 
pelos profissionais de saúde, o que os coloca num ciclo permanente de ensinar e de aprender. As políticas públicas de saúde demandam a formação adequada dos recursos humanos, tema instigante pelas transformações que requer para a consolidação do Sistema Único de Saúde (SUS) (Celedônio, Jorge, dos Santos, de Freitas, \& de Aquino, 2012).

A formação na modalidade "residência em saúde" é considerada uma modalidade de treinamento em serviço, que tem como base a aprendizagem pela prática cotidiana, a partir da exposição a situações próprias para a formação, que representam momentos do dia a dia profissional, planeados de forma didática. Além de contribuir para a construção ideológica pautada pela ética e pela identidade profissional, a sua compreensão não se limita à de um projeto educacional de especialização isolado, mas também de formação de força de trabalho nas instituições mantedoras de programas e de um espaço de política de saúde (Feijó, Fakhouri Filho, Nunes, \& Augusto, 2019).

$\mathrm{Na}$ perspectiva teórica de Sacristán (2013), o papel do professor é determinante para a formação crítica do indivíduo. Ressalta que toda experiência pedagógica supõe o propósito de mediação, correção e estímulo da experiência do encontro entre um sujeito que exerce uma série de funções sobre o sujeito que desenvolve diversas capacidades, atuando para sua transformação e enriquecimento. Esse encontro, se adequadamente mediado e motivado, pode ser significativo, relevante e desafiador.

O cenário de formação é um desafio pedagógico a ser enfrentado pelos programas de residência. Assim, a construção das transformações esperadas implica formulação e utilização de um conjunto de estratégias para reflexão e produção de desconfortos, mobilização e construção de tecnologias para responder às novas situações, pois não há um elemento isolado que, incentivado, dê conta de desencadear as mudanças necessárias. Por isso, não se pode pensar em mudanças na formação sem a produção concomitante de muitos processos de ação nos territórios e nas unidades construídas de modo compartilhado (Feuerwerker, 2014). No cenário da produção de saúde, o sistema de saúde brasileiro ainda tem grandes desafios para a oferta de atenção integral e de qualidade às mulheres. Todavia se identificam cenários com potencial de agir sinergicamente para uma efetiva mudança na atenção ao parto e nascimento com a implementação de boas práticas perinatais como política pública (Carvalho, Amorim, Santana, \& Göttems, 2019).

Em vista disso, verifica-se a importância dos projetos pedagógicos como guia dos processos de ensino-aprendizagem nos cenários de ensino-serviço. Perante esse contexto e a 
premissa de que os cursos de residência obstétrica se propõem a formar profissionais preparados para atender à mulher de forma integral e humanizada, alinhados às políticas norteadoras da promoção do parto e nascimento e orientados pelos princípios e diretrizes do SUS, este estudo tem como objetivo verificar se os projetos pedagógicos dos cursos de residência obstétrica, de médicos(as) e enfermeiros(as), dos serviços de saúde da Secretaria de Saúde do Distrito Federal contemplam as recomendações das diretrizes nacionais e internacionais de atenção ao parto normal.

\section{METODOLOGIA}

Trata-se de um estudo descritivo, exploratório, com triangulação de dados, de abordagem qualitativa, por meio de análise documental e análise de conteúdo. A análise documental consiste em buscar identificações factuais nos documentos a partir de questões e hipóteses de interesse (Lüdke \& André, 1986). Na análise dos documentos, espera-se responder a seguinte questão: As recomendações das diretrizes nacionais e internacionais de atenção ao parto estão contempladas textualmente nos projetos pedagógicos dos cursos de residência em obstetrícia? E, nas entrevistas, fez-se o questionamento: Como as boas práticas de atenção ao parto e nascimento são abordadas durante o programa de residência (O que é ensinado? Como é ensinado?)? Que práticas demonstrativamente úteis são utilizadas pelos residentes no trabalho de parto?

A pesquisa qualitativa responde a questões muito particulares. Preocupa-se com um nível de realidade que não pode ser quantificado (Minayo, 2014). A triangulação de dados surge como uma estratégia de diálogo entre áreas distintas de conhecimento, capaz de viabilizar o entrelaçamento entre a teoria e a prática e de agregar múltiplas fontes de evidência em relação a um mesmo conjunto de questões, visando corroborar a mesma descoberta (Ribeiro, Brandão, \& Costa, 2016).

Esta pesquisa foi realizada nos serviços de obstetrícia da Secretaria de Estado de Saúde do Distrito Federal. Os projetos pedagógicos dos cursos de residência de Enfermagem e Medicina foram os documentos analisados neste estudo. Complementarmente, realizaramse entrevistas individuais com 35 preceptores. A supervisão do processo de formação é efetuada atualmente por 15 enfermeiros e 64 médicos da rede. Foram convidados preceptores de todos os serviços, o que caracteriza a amplitude da pesquisa. Participaram 35 preceptores, sendo 14 enfermeiros e 21 médicos. A amostra foi não probabilística e adotou critérios de conveniência (Vergara, 2009). Utilizou-se, como parâmetro da saturação 
amostral, o esgotamento de novos assuntos no discurso dos respondentes (Flick, 2009). Os critérios de inclusão foram elaborados para cada uma das etapas deste estudo - em relação aos documentos: projeto pedagógico dos cursos de residência em medicina e enfermagem dos oito hospitais onde se realizam as atividades práticas; em relação aos preceptores: ser médico ou enfermeiro, atuar como preceptor e desejar participar na pesquisa, voluntariamente. Os projetos foram obtidos a partir de agosto de 2017, mediante solicitação por correspondência eletrônica (e-mail) para os coordenadores das Comissões de Residência Médica (COREME) e para a Coordenadora da Residência em Enfermagem Obstétrica. Para análise dos projetos pedagógicos, empregaram-se técnicas usuais de análise de conteúdo a fim de decifrar, em cada texto, o núcleo emergente que atendesse ao propósito da pesquisa. Essa etapa consistiu num processo de codificação, interpretação e de inferências sobre as informações contidas nos projetos (Minayo, 2014).

As entrevistas com os preceptores dos cursos de residência foram realizadas entre março e junho de 2018 e foram analisadas por meio da análise de conteúdo, que aconteceu em três etapas. A primeira etapa foi realizada por meio da leitura flutuante do material transcrito, de forma a permitir apreender e organizar, aspectos importantes para as fases seguintes da análise; a segunda etapa consistiu na exploração do material e no agrupamento do texto em categorias pré-estabelecidas; e a terceira etapa, o tratamento e a interpretação dos dados, permitiu a realização de inferências dos conteúdos obtidos na entrevista (Bardin, 2011; Minayo, 2014). Este estudo seguiu as determinações da Resolução no 466, de 12 de dezembro de 2012, do Conselho Nacional de Saúde, que dispõe sobre diretrizes e normas regulamentadoras de pesquisas envolvendo seres humanos. O projeto foi aprovado pelo Comitê de Ética em Pesquisa da Secretaria de Estado de Saúde do Distrito Federal CEP/SES/DF sob o Parecer № 2.166.900 de 10 de julho de 2017.

\section{RESULTADOS E DISCUSSÃO}

\subsection{Análise dos Projetos Pedagógicos dos cursos}

O projeto político-pedagógico implica em estratégias e propostas práticas de ação. Para educar não basta indicar um horizonte e um caminho para se chegar lá. É preciso indicar como se chega lá e fazer o caminho juntos. O projeto deve indicar grandes perspectivas, quais os valores que orientam a ação educativa, as ideologias em jogo, uma discussão do contexto local, nacional e internacional. Ele deve retratar as aspirações, ideais e anseios da 
comunidade acadêmica. Projetar é escolher, decidir. E a escolha, a decisão, são categorias pedagógicas essenciais ao ato educativo (Gadotti, 2016).

$\mathrm{Na}$ modalidade de ensino da residência, onde $80 \%$ a $90 \%$ do curso se dá no cenário da prática assistencial, destaca-se a relevância do planejamento das ações do projeto pedagógico, não apenas sobre o que é ensinado, mas como é ensinado. Para Freire (2018), nas condições de verdadeira aprendizagem, os educandos vão se transformando em reais sujeitos da construção e da reconstrução do saber ensinado, ao lado do preceptor, igualmente sujeito do processo. Nos projetos político-pedagógicos buscou-se verificar se as políticas públicas relacionadas ao parto e nascimento estavam contempladas, conforme as recomendações do Ministério da Saúde (MS) e da Organização Mundial da Saúde (OMS). Inicialmente foi feito uma organização do material, leitura dos projetos buscando sínteses coincidentes e divergentes de ideias, e posteriormente foram definidas as seguintes categorias de análise: Aspectos estruturais dos Projetos; Perfil de Competências do Egresso e, por último, Políticas públicas norteadoras do parto normal.

Quadro 1. Projetos político-pedagógicos do Programa de Residência Médica.

Brasília/DF, 2019.

\begin{tabular}{|l|l|}
\hline \multicolumn{1}{|c|}{ Aspectos estruturais } & \multicolumn{1}{c|}{ Perfil de competência dos egressos } \\
\hline Diretrizes pedagógicas & $\begin{array}{l}\text { Conhecer e interpretar os principais aspectos epidemiológicos, demográficos } \\
\text { e sócio-econômico-culturais que interferem na saúde das mulheres }\end{array}$ \\
\hline Objetivos gerais e específicos & $\begin{array}{l}\text { Desenvolver conhecimentos para o adequado entendimento da relação } \\
\text { entre alterações psíquicas e distúrbios tocoginecológicos }\end{array}$ \\
\hline Processo avaliativo discente & $\begin{array}{l}\text { Praticar a assistência pré-natal de baixo e alto risco; eapacitar na } \\
\text { prevenção, diagnóstico e tratamento das principais intercorrências clínicas e } \\
\text { obstétricas }\end{array}$ \\
\hline Autoavaliação do programa & $\begin{array}{l}\text { Desenvolver capacidade de julgamento e discernimento para indicação de } \\
\text { exames e tratamentos eficazes e eficientes }\end{array}$ \\
\hline Corpo docente/ano & $\begin{array}{l}\text { Capacidade comunicativa com pacientes, colegas, profissionais da área e } \\
\text { demais pessoas envolvidas com o tratamento }\end{array}$ \\
\hline Programa teórico e prático & Capacidade de trabalho em equipe de forma harmoniosa. \\
\hline Duração e carga horária total & Observância dos princípios éticos \\
\hline Atribuições do supervisor & Compromisso e responsabilidade profissional \\
\hline Atribuições do preceptor & $\begin{array}{l}\text { Formar especialistas com conhecimentos, habilidades e competências para } \\
\text { a atuação dentro da rede do SUS e da saúde suplementar }\end{array}$ \\
\hline Perfil e competência do egresso & Atendimento integral da saúde da mulher de forma humanizada \\
\hline $\begin{array}{l}\text { Critérios para a conclusão do PRM } \\
\text { e para certificação }\end{array}$ & Promover maior interação ensino-serviço. \\
\hline
\end{tabular}

O Quadro 1 apresenta as duas categorias identificadas nos Projetos da Residência Médica, seguidas das unidades de registro. Os aspectos estruturantes do projeto pedagógico, bem como as competências a serem desenvolvidas pelos egressos são similares nos projetos dos programas de residência médica analisados. Para além dos conteúdos, é preciso insistir que os significados dos objetivos educacionais não podem estar circunscritos aos conteúdos 
dos limites estabelecidos pelas tradições acumuladas, mas que podem e devem ser revisados e modificados (Sacristán, 2013).

Embora o Brasil tenha editado políticas, programas e estratégias, desde a década de 1980, a implementação nos serviços de saúde ainda é uma agenda incompleta. Dos 3 milhões de partos ocorridos anualmente, 55,5\% são por cesariana, apesar da crescente criação de Centros de Parto Normal e de movimentos em prol do parto normal terem ganhado força (Mascarenhas, 2019).

Quadro 2. Projeto político-pedagógico do Programa de Residência em Enfermagem Obstétrica. Brasília, 2019.

\begin{tabular}{|c|c|c|}
\hline Aspectos estruturais & Perfil de competências dos egressos & $\begin{array}{l}\text { Políticas públicas } \\
\text { norteadoras da promoção } \\
\text { do parto normal }\end{array}$ \\
\hline Diretrizes pedagógicas & $\begin{array}{l}\text { Conhecer e aplicar as Políticas Públicas de } \\
\text { Saúde Materna e Perinatal }\end{array}$ & $\begin{array}{l}\text { Utilizar as referências } \\
\text { norteadoras da promoção do } \\
\text { parto normal. }\end{array}$ \\
\hline $\begin{array}{l}\text { Objetivos gerais e } \\
\text { específicos }\end{array}$ & $\begin{array}{l}\text { Prestar assistência e cuidados de enfermagem } \\
\text { de forma sistematizada, integral e humanizada à } \\
\text { mulher no ciclo gravídico-puerperal, bem como } \\
\text { a seu recém-nascido e familiares, no âmbito da } \\
\text { assistência primária e secundária }\end{array}$ & $\begin{array}{l}\text { Programa de Atenção Integral } \\
\text { à Saúde da Mulher }\end{array}$ \\
\hline $\begin{array}{l}\text { Processo avaliativo } \\
\text { discente }\end{array}$ & $\begin{array}{l}\text { Assistir a mulher em situações de emergência } \\
\text { obstétrica }\end{array}$ & $\begin{array}{l}\text { Relatório Técnico da OMS/MS } \\
\text { - Assistência ao Parto } \\
\text { Normal: um guia prático }\end{array}$ \\
\hline $\begin{array}{l}\text { Autoavaliação do } \\
\text { programa }\end{array}$ & $\begin{array}{l}\text { Assistir a mulher e seus familiares em situações } \\
\text { de violência }\end{array}$ & $\begin{array}{l}\text { Programa de Humanização do } \\
\text { Pré-natal e Nascimento }\end{array}$ \\
\hline Corpo docente/ano & $\begin{array}{l}\text { Assistir à mulher, no ciclo gravídico-puerperal, } \\
\text { com intercorrências clínicas e patologias } \\
\text { obstétricas }\end{array}$ & $\begin{array}{l}\text { Política Nacional de Atenção } \\
\text { Integral à Saúde da Mulher }\end{array}$ \\
\hline $\begin{array}{l}\text { Programa teórico e } \\
\text { prático }\end{array}$ & $\begin{array}{l}\text { Desenvolver capacidade crítico-reflexiva } \\
\text { relacionada à prática profissional }\end{array}$ & Programa da Rede Cegonha. \\
\hline $\begin{array}{l}\text { Duração e carga horária } \\
\text { total }\end{array}$ & $\begin{array}{l}\text { Planejar e executar programas educativos para } \\
\text { os usuários e equipe de enfermagem }\end{array}$ & \\
\hline $\begin{array}{l}\text { Perfil e competência do } \\
\text { egresso }\end{array}$ & $\begin{array}{l}\text { Prestar cuidados baseados em evidências } \\
\text { científicas, primando pelos princípios éticos da } \\
\text { profissão }\end{array}$ & \\
\hline & $\begin{array}{l}\text { Estimular a promoção de mudanças de } \\
\text { paradigmas assistenciais, voltados à prática da } \\
\text { saúde baseada em evidências científicas }\end{array}$ & \\
\hline
\end{tabular}

O Quadro 2 apresenta as três categorias identificadas, seguidas das unidades de registro. $\mathrm{O}$ projeto político-pedagógico do curso de residência de enfermagem traz, de forma contextual, todos os programas e políticas do Ministério da Saúde para estimular a promoção do parto normal e humanizado. Faz referência ao Programa de Assistência Integral à Saúde da Mulher (Brasil, 1984), que foi criado com o objetivo de melhorar a saúde da população feminina, abrangendo as necessidades e demandas em todas as fases e ciclo de vida, reduzindo a morbimortalidade. Faz referência ao Relatório técnico "Assistência ao parto normal: um guia prático" (OMS, 1996), que classifica as práticas segundo utilidade, eficácia 
e risco, para orientar a conduta do profissional no manejo do trabalho de parto e parto. A partir desse documento, foi possível a provocação dos profissionais para repensar o parto como um evento que transcende aspectos estritamente biológicos. O Programa de Humanização do Pré-natal e Nascimento (Brasil, 2000), que tem como objetivo reorganizar a assistência ao ciclo gravídico-puerperal, ampliar o acesso e garantir a qualidade da assistência, também é citado. Traz a Rede Cegonha (Brasil, 2011) como estratégia inovadora do MS que tem como prioridade a redução da mortalidade materna e infantil, por meio da ampliação e qualificação das ações e serviços de saúde, do combate à violência obstétrica, da oferta das boas práticas e da redução da medicalização e mercantilização do parto. Como a última atualização do projeto político-pedagógico do curso de enfermagem foi em 2016, não contempla, portanto, a Diretriz Nacional de Assistência ao Parto Normal (Brasil, 2017), que é o mais novo documento do MS. Essa diretriz foi elaborada por um grupo multiprofissional e multidisciplinar que visa orientar as mulheres brasileiras, os profissionais de saúde e os gestores, nos âmbitos público ou privado, sobre importantes questões relacionadas às vias de parto, indicações e condutas, baseadas nas melhores evidências científicas disponíveis. Entre o escopo e as finalidades almejadas, propõe-se a promover modificações na prática clínica, uniformizar e padronizar as práticas utilizadas na assistência ao parto normal; diminuir a variabilidade injustificada de condutas entre os profissionais; reduzir intervenções desnecessárias; difundir e aumentar as melhores práticas baseadas em evidências e fazer recomendações contra ou a favor de algumas práticas no processo de decisão e condução da assistência ao parto normal. O Projeto pedagógico do curso de enfermagem traz conteúdos que corroboram a promoção de mudanças de paradigmas assistenciais, voltados à prática da saúde baseada em evidências científicas. Para Leal (2019), a presença da enfermeira obstetra na equipe de atenção ao parto está associada a mais acesso às boas práticas recomendadas pela OMS, consequentemente a melhores resultados no trabalho de parto e parto; redução de intervenções desnecessárias, inclusive cesarianas; aumento da satisfação das mulheres com o atendimento recebido e melhores resultados perinatais.

\subsection{Análise das entrevistas com os preceptores}

Dentre os participantes ( $n=35$ ), $40 \%$ são enfermeiros e $60 \%$, médicos. Com relação ao sexo, $74,28 \%$ são do sexo feminino e $25,72 \%$ do sexo masculino. A média de idade foi de 45 anos, sendo a média do tempo de curso, de 20 anos e do tempo de atuação na preceptoria, 8 anos. Dentro do objetivo proposto, visando complementar as informações dos documentos 
investigados, buscou-se verificar na fala dos preceptores como as boas práticas são discutidas no programa de residência, o que é abordado, como é abordado, e quais práticas demonstrativamente úteis são mais utilizadas no serviço. Os participantes foram referenciados pela categoria profissional ( $M=$ médico e $E=e n f e r m e i r o)$, seguido de número cardinal ( $E 1$ ou $M 1$, e assim sucessivamente).

Quadro 3. Ensino das boas práticas no programa de residência, segundo os preceptores.

Brasília, 2019

\begin{tabular}{|c|c|}
\hline M5 & $\begin{array}{l}\text { E discutido não só o que está nos livros textos como também em relação às diretrizes das boas práticas em } \\
\text { relação ao parto, às recomendações da OMS de } 1996 \text { e às diretrizes atuais do Ministério da Saúde. }\end{array}$ \\
\hline M9 & $\begin{array}{l}\text { É visto no programa teórico-prático juntamente com os demais itens que constam da programação teórica e } \\
\text { é ensinado na prática do dia a dia. }\end{array}$ \\
\hline M14 & $\begin{array}{l}\text { Todo começo do ano tem as aulas teóricas, então no começo de cada ciclo que entram os residentes, eles } \\
\text { têm as aulas teóricas, e uma das aulas é sobre as boas práticas, então eles têm aula teórica sobre boas } \\
\text { práticas em obstetrícia, nascimento, puerpério, isso todo ano é feito, e na parte prática é empenho do } \\
\text { residente e espelho do preceptor. }\end{array}$ \\
\hline M17 & $\begin{array}{l}\text { Então, nós discutimos isso de forma teórica, com artigos publicados, as recomendações do Ministério da } \\
\text { Saúde (MS), os guias do MS e, durante o plantão, isso é colocado em prática, tem sido feito da maneira } \\
\text { como é recomendado. }\end{array}$ \\
\hline E4 & $\begin{array}{l}\text { Hoje em dia, a prática já condiz mais com a teoria e desde o início, desde políticas públicas do primeiro } \\
\text { módulo, a gente já traz os protocolos, as evidências e as reflexões da prática continuam também. Então, } \\
\text { sempre baseados em evidências, não só aquele modelo de repetição, do porquê. Então, a residência é } \\
\text { pautada, ela é sedimentada nessas boas práticas. }\end{array}$ \\
\hline E5 & $\begin{array}{l}\text { A formação na residência em enfermagem é voltada pra fazer a diferença na atenção à mulher e à criança, } \\
\text { então na inserção deste enfermeiro no serviço é condicionado às boas práticas sabidamente por pesquisas e } \\
\text { evidências científicas ser o melhor para a mãe e o bebê nesse momento. }\end{array}$ \\
\hline E6 & $\begin{array}{l}\text { Olha, a residência ela veio para realmente mudar o que era errado e a gente inserir as boas práticas no } \\
\text { parto. Uma coisa mais importante da residência não é apenas o conhecimento, nem pegar menino, é a gente } \\
\text { fazer aquilo que o OMS já orienta há muito tempo, e deixar a mulher parir no seu tempo, a gente tendo o } \\
\text { conhecimento, tendo todo o conhecimento técnico-científico, mas a gente respeitar essa mulher. }\end{array}$ \\
\hline E8 & $\begin{array}{l}\text { Então, na verdade o que a gente busca é um alinhamento entre o conteúdo teórico e a prática. Na parte } \\
\text { teórica é muito tranquilo porque o nosso cuidado, o nosso ensino, ele já é embasado em evidências né, } \\
\text { então a gente pega o que tem mais de recente pra respaldar né, e pra gente passar isso para o residente. }\end{array}$ \\
\hline E13 & $\begin{array}{l}\text { Durante o desenvolvimento da residência, eles têm uma oficina, tem tutoria sobre boas práticas, seria a parte } \\
\text { teórica e aqui eles vêm para aplicar, seria a parte prática do que eles já aprenderam na teoria, então eles } \\
\text { vêm pra cá, atendemos pacientes, aí são orientados por nós com todos os recursos e mecanismos } \\
\text { facilitadores das boas práticas e eles aprendem junto com a gente, assistindo a gente a fazer e fazendo junto } \\
\text { com a gente essas boas práticas. }\end{array}$ \\
\hline
\end{tabular}

O quadro 3 é um apanhado das respostas relacionadas a como as boas práticas são abordadas durante o programa de residência ( $\mathrm{Q}$ que é ensinado? Como é ensinado?). Buscou-se fazer uma interação entre o conteúdo manifesto nos documentos com o conteúdo das falas dos respondentes. Segundo os preceptores, eles ensinam as boas práticas recomendadas pelas diretrizes nacionais e internacionais de assistência ao parto normal e buscam o alinhamento entre a teoria e a prática desde a sala de aula até os cenários de práticas. No entanto, quando se analisam os projetos da medicina, verificam-se lacunas quanto à descrição de políticas públicas com referências à promoção das boas práticas no parto normal. 
Considera-se que parte dos preceptores de hoje ainda são fruto do modelo de formação antigo que viveram como alunos-residentes e reproduzem os modelos biomédicos e hospitalocêntricos, fugindo à promoção da prática problematizadora que leva o aluno/residente a questionar e refletir sobre os fatos com os quais deparam, levando à formação de uma consciência crítica. E pensar não é somente "raciocinar" ou "calcular" ou "argumentar", como nos tem sido ensinado, mas é sobretudo dar sentido ao que somos e ao que nos acontece (Freitas et al., 2016).

A necessidade de mudança na formação profissional com sustentação teórico-prática do cuidado obstétrico humanizado é uma das estratégias pensadas pela Rede Cegonha (Brasil, 2011) para a implementação de uma assistência que privilegie o bem-estar da parturiente e do recém-nascido. Além disso, tal modelo busca ser o menos invasivo possível, considerando tanto os processos fisiológicos quanto os psicológicos e o contexto sociocultural. Considera-se que reaprender é muito mais difícil que aprender na formação inicial, e que a mudança no modelo de atenção ao parto pode se dar por meio da formação adequada de novos profissionais, forjados na expertise das boas práticas obstétricas. Com base nisso, é fundamental refletir sobre as perspectivas de formação que transcendam as abordagens de natureza técnico-pedagógica, pois o processo educativo é essencialmente social, relacional, comunicativo e político (Paula, 2018).

Práticas demonstrativamente úteis mais utilizadas no serviço, segundo os preceptores a Tabela 1 representa a quantificação das respostas em ordem de recorrência sobre as principais práticas demonstrativamente úteis citadas pelos respondentes. Os métodos não farmacológicos de alívio da dor foram os mais citados, tanto pelas enfermeiras como pelos médicos. Eles citaram a massagem, os exercícios respiratórios, o banho morno relaxante, o uso da bola e do cavalinho como práticas mais comuns utilizadas no manejo do parto.

Tabela 1. Principais práticas demonstrativamente úteis citadas pelos participantes.

Brasília, 2019

\begin{tabular}{|c|c|c|c|c|}
\hline \multirow{3}{*}{ Práticas demonstrativamente úteis } & \multicolumn{4}{|c|}{ Frequência } \\
\hline & \multicolumn{2}{|c|}{ Enfermeiros } & \multicolumn{2}{|c|}{ Médicos } \\
\hline & № & $\%$ & № & $\%$ \\
\hline Métodos não farmacológicos de alívio da dor & 14 & $100,0 \%$ & 13 & $61,9 \%$ \\
\hline Presença do acompanhante & 11 & $78,5 \%$ & 16 & $76,1 \%$ \\
\hline Deambülação & 13 & $92,8 \%$ & 8 & $38,0 \%$ \\
\hline Liberdade de posição e movimento & 11 & $78,5 \%$ & 8 & $38,0 \%$ \\
\hline $\begin{array}{l}\text { Clampeamento oportuno do cordão/contato pele a pele/ } \\
\text { amamentação na primeira hora pós-parto }\end{array}$ & 5 & $35,7 \%$ & 8 & $38,0 \%$ \\
\hline Posições alternativas no parto & 8 & $57,1 \%$ & 4 & $19,0 \%$ \\
\hline Alimentação e líquidos durante o trabalho de parto & 5 & $35,7 \%$ & 9 & $42,8 \%$ \\
\hline Apoio/Suporte & 6 & $42,8 \%$ & 3 & $14,3 \%$ \\
\hline
\end{tabular}


A presença do acompanhante foi a segunda prática mais citada pelos respondentes. $O$ acompanhante é uma personagem indispensável durante o trabalho de parto (TP) e parto normal, pois fornece à mulher apoio emocional, tranquilidade, segurança, conforto, e assim minimiza as suas preocupações e medos e torna o nascimento humanizado (Dias, Ferreira, Martins, de Jesus Nunes, \& Alves, 2018). A deambulação, método não farmacológico que promove uma tolerância maior da parturiente quanto à dor no trabalho de parto, foi a terceira prática mais citada (Araújo et al., 2018).

Foram citadas também, a liberdade de posição e movimento, clampagem oportuna do cordão, contato pele a pele, amamentação na primeira hora pós-parto, posições alternativas no parto, utilização de alimentos e líquidos durante o TP e parto e o apoio/suporte emocional. E, assim, os preceptores de ambos os programas de residência mostram na prática e discutem na teoria a importância da utilização das práticas demonstrativamente úteis para desfechos perinatais positivos.

\section{CONCLUSÕES}

Observou-se que o projeto político-pedagógico do curso de enfermagem contempla as recomendações do MS e da OMS. O curso tem como eixo norteador a ideologia do cuidado centrado na mulher, o incentivo à utilização das boas práticas obstétricas, a redução das intervenções desnecessárias, a desmedicalização da saúde, o fomento à autonomia e o protagonismo da mulher. Concebe a assistência de enfermagem como uma construção compartilhada, na qual as preceptoras se orientam pelo princípio da não intervenção.

Os projetos político-pedagógicos da residência médica ainda não contemplam de forma textual as recomendações do MS e da OMS relacionadas à temática das boas práticas no parto e nascimento. Considerando a interação entre gestão, ensino, serviço e atenção e as políticas intergovernamentais articuladas entre Ministério da Saúde e Ministério de Educação, recomenda-se que os conteúdos dos projetos pedagógicos dos cursos da residência sejam revistos, visando corroborar a formação adequada dos futuros profissionais.

A investigação qualitativa foi determinante para o êxito deste estudo, pois consegue aprofundar a análise e desvendar o conteúdo latente, revelando a necessidade de fortalecer o processo de ensino, cuidado e gestão na prática da preceptoria. Para tanto, faz-se necessário que os serviços de saúde pesquisados possam viabilizar a reformulação dos 
projetos pedagógicos de forma a contemplar as recomendações do MS e da OMS; que os preceptores, enquanto sujeitos ativos de mudança, possam colaborar na discussão e reestruturação dos projetos, com vistas a promover a formação de profissionais com formação crítica, reflexiva, comprometidos com a vida e com a saúde da mulher e da criança. As limitações desta pesquisa estão relacionadas à coleta de dados dos projetos pedagógicos de médicos e enfermeiros apenas (curso de pós-graduação lato sensu), não permitindo a análise de projetos pedagógicos de outras especialidades que, de alguma forma, façam interface com o binômio mãe-filho. Considera-se que a investigação qualitativa é relevante para continuar a problematizar as boas práticas em obstetrícia, sobretudo no parto normal, as quais podem ser aprofundadas por pesquisas futuras.

\section{REFERÊNCIAS}

Aragão, H. T., Vieira, S. S., de Santana Fernandes, É. T., \& da Silva, G. M. (2017). Trabalho de parto e os métodos não farmacológicos para alívio da dor: revisão integrativa. In Congresso Internacional de Enfermagem (v. 1, n. 1).

Araújo, A. D. S. C., de Medeiros Correia, A., Rodrigues, D. P., Lima, L. M., de Santana Gonçalves, S., \& da Silva Viana, A. P. (2018). Non-pharmacological methods in home birth. Journal of Nursing UFPE on line, 12(4), 1091-1096.

Bardin, L. (2011). Análise de conteúdo. São Paulo: Edições 70.

Brasil. (1984). Ministério da Saúde. Programa de Assistência Integral à Saúde da Mulher: bases de ação programática. Brasília: Centro de Documentação do Ministério da Saúde/MS.

Brasil. (2000). Ministério da Saúde. Portaria n. 569, de 1o de junho de 2000. Dispõe sobre o Programa de Humanização ao Pré-Natal e Nascimento no âmbito do Sistema Único de Saúde. Diário Oficial da União, Brasília-DF, 8 jun. 2000, Seção 1:4

Brasil. (2011). Ministério da Saúde. Portaria no 1.459 de 24 de junho de 2011. Institui no âmbito do Sistema Único de Saúde - SUS - a Rede Cegonha. Brasília: Ministério da Saúde.

Brasil. (2017). Ministério da Saúde. Secretaria de Ciência, Tecnologia e Insumos Estratégicos. Departamento de Gestão e Incorporação de Tecnologias em Saúde. Diretrizes Nacionais de Assistência ao Parto Normal: versão resumida. Brasília: Ministério da Saúde.

Carvalho, E. M. P.de, Amorim, F. F., Santana, L. A., \& Göttems, L. B. D. (2019). Avaliação das boas práticas de atenção ao parto por profissionais dos hospitais públicos do Distrito Federal, Brasil. Ciência \& Saúde Coletiva, 24(6), 2135-2145.

Celedônio, R. M., Jorge, M. S. B., dos Santos, D. C. M., de Freitas, C. H. A., \& de Aquino, F. O. T. P. (2012). Políticas de educação permanente e formação em saúde: uma análise documental. Revista da Rede de Enfermagem do Nordeste, 13(5), 1100-1110.

Dias, E. G., Ferreira, A. R. M., Martins, A. M. C., de Jesus Nunes, M. M., \& Alves, J. C. S. (2018). Eficiência de métodos não farmacológicos para alívio da dor no trabalho de parto normal. Enfermagem em Foco, 9(2), 35-39.

Feuerwerker, L. C. M. (2014). Micropolítica e saúde: produção do cuidado, gestão e formação. Porto Alegre: Rede Unida. 
Feijó, L. P., Fakhouri Filho, S. A., Nunes, M. D. P. T., \& Augusto, K. L. (2019). Resident as a Teacher: an Introduction to Teaching. Revista Brasileira de Educação Médica, 43(2), 225-230.

Flick, U. (2009). Introdução à pesquisa qualitativa. Porto Alegre: Artmed.

Freire, P. (2018). Pedagogia da autonomia: saberes necessários à pratica educativa. Rio de Janeiro: Paz e Terra.

Freitas, D. A., Santos, E. M. S., Lima, L. V. S., Miranda, L. N., Vasconcelos, E. L., \& Nagliate, P. C. (2016). Saberes docentes sobre processo ensino-aprendizagem e sua importância para a formação profissional em saúde. Interface (Botucatu), 20(57), 437-448.

Gadotti, M. (2016). Dimensão política do projeto pedagógico da escola. Secretaria de Estado da Educação de Minas Gerais, Diretoria de Capacitação de Recursos Humanos PROCAD - Projeto de Capacitação de Dirigentes Fase Escola Sagarana. Acervo Moacir Gadotti.

Sacristán, J. G. (2013). Saberes e incertezas sobre o currículo. Porto Alegre: Penso.

Leal, M. D. C., Bittencourt, S. D. A., Esteves-Pereira, A. P., Ayres, B. V. D. S., Silva, L. B. R. A. D., Thomaz, E. B. A. F., ... \& Domingues, R. M. S. M. (2019). Progress in childbirth care in Brazil: preliminary results of two evaluation studies. Cadernos de Saúde Pública, 35, e00223018.

Lüdke, M., \& André, M. (1986). Pesquisa em educação: abordagens qualitativas. São Paulo: EPU.

Mascarenhas, V. H. A., Lima, T. R.,, Silva, F. M. D. e, Negreiros, F. S., Santos, J. D. M.,, Moura, M. A. P., Gouveia, M. T. O., \& Jorge, H. M. F. (2019). Evidências científicas sobre métodos não farmacológicos para alívio a dor do parto. Acta Paulista de Enfermagem, 32(3), 350-357.

Minayo, M. C. D. S. (2014). O desafio do conhecimento: pesquisa qualitativa em saúde. 4. ed. São Paulo: Hucitec.

Organização Mundial de Saúde (1996). Saúde Materna e Neonatal. Unidade Maternidade Segura, Saúde Reprodutiva e da Família. Assistência ao parto normal: guia prático. Genebra (SW): OMS.

Paula, L. C., \& de Mello, R. R. (2018). A práxis histórica de Paulo Freire como fundamentação para as pesquisas sobre formação de educadores. Reflexão e Ação, 26(1), 06-23.

Ribeiro, J. M., Brandão, C., \& Costa, A. P. (2016). Metodologia de estudo de caso em saúde: contributos para a sua qualidade. In: Oliveira, E., Barros, N., Silva, R., organizadores. Investigação qualitativa em saúde: conhecimento e aplicabilidade. Oliveira de Azeméis: Ludomedia. p. 143-160.

Vergara, S. C. (2009). Projetos e relatórios de pesquisa em Administração. São Paulo: Atlas. 